\title{
SIMULATION OF METALLIC PHOTONIC BANDGAP STRUCTURES FOR ACCELERATOR APPLICATIONS
}

\author{
E.I. Smirnova*, C. Chen, M.A. Shapiro, R.J. Temkin, \\ Plasma Science and Fusion Center, MIT, Cambridge, MA 02139, USA
}

\begin{abstract}
Use of microwave photonic bandgap (PBG) structures is a promising approach to improving the performance of rf accelerator structures in terms of better control of wakefields. We have developed the Photonic Band Gap Structure Simulation (PBGSS) code to study bulk properties of electromagnetic $\mathrm{TE}$ and $\mathrm{TM}$ wave propagation in two-dimensional (2D) PBG structures. The eigenmodes are calculated and global photonic bandgaps are determined. Rf accelerator applications of PBG cavities are discussed.
\end{abstract}

\section{INTRODUCTION}

Since the pioneering work of Yablonovitch in the late 1980s [1], PBG structures have emerged as a growth area for research and development [2-5]. While initial studies of $\mathrm{PBG}$ structures were primarily focused on dielectric PBG structures [1-3], metallic PBG structures [4-6], as well as dielectric-metallic hybrids, have received considerable attention recently, because of their applications in rf accelerators and high-power microwave electronics [5-7].

Two important aspects need to be studied in order to facilitate the design of metallic PBG-based devices. One involves the wave propagation in bulk of the PBG structure, and the other concerns mode confinement in a metallic PBG cavity. For analyses of metallic PBG cavities formed by single or multiple defects in PBG structure, finite-element codes such as HFSS [8] are ideally suited. We developed a code to study the dispersion properties of the wave propagating in the infinite metallic PBG structure.

We solve an important and computationally challenging problem of calculation of the global photonic band gaps in metallic lattices [9]. While a number of papers dealt with the global band gaps in dielectric lattices [2,3], only the lowest gap for a TM mode in a square metallic lattice was calculated [4]. We extend the calculation to wide range of frequencies and to both square and triangular lattices.

\section{PBGSS ALGORITHM DESCRIPTION}

We consider the square and triangular lattices shown in Fig. 1 ( on the figure $a$ is the radius of the conducting cylinder, $b$ is the lattice spacing). The conductivity profile in the lattice satisfies the periodic condition

$$
\sigma\left(\mathbf{x}_{\perp}+\mathbf{T}_{m n}\right)=\sigma\left(\mathbf{x}_{\perp}\right)
$$
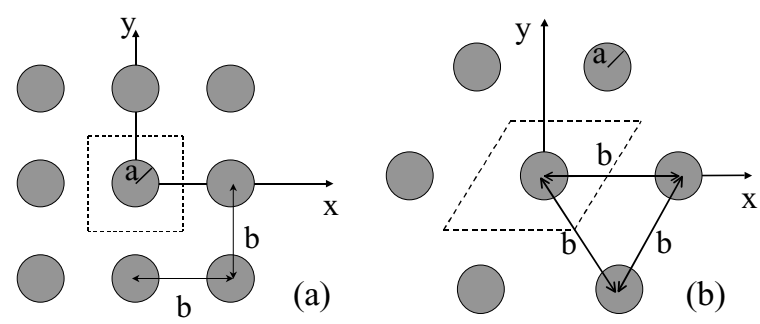

Figure 1: Scheme of PBG structure representing (a) square lattice and (b) triangular lattice of perfectly conducting cylinders.

where $\mathbf{x}_{\perp}=x \hat{\mathbf{e}}_{x}+y \hat{\mathbf{e}}_{y}$ is the transverse displacement, $m$ and $n$ are integers, and $\mathbf{T}_{m n}$ is one of the lattice periodicity vectors.

The wave field in 2D PBG structure can be decomposed into two independent classes of modes: the transverse electric (TE) mode and the transverse magnetic (TM) mode. All the field components in the TM (TE) modes can be expressed through the axial component of the electric (magnetic) field, which we will further denote by $\psi$. Since the system is homogeneous along the $z$-axis, we take the Fourier transform of $\psi$ in axial coordinate $z$ and time $t$. The Helmholtz equation for $\psi\left(\mathbf{x}_{\perp}, k_{z}, \omega\right)$ follows from the Maxwell equations

$$
\nabla_{\perp}^{2} \psi=\left(k_{z}^{2}-\frac{\omega^{2}}{c^{2}}\right) \psi
$$

The boundary conditions on the surfaces $S$ of the conducting poles are $\left.\psi\right|_{S}=0$ for the TM mode and $\left.\frac{\partial \psi}{\partial \mathbf{n}}\right|_{S}=0$ for the TE mode ( $\mathbf{n}$ is the normal vector to the pole surface). The discrete translational symmetry of the conductivity profile allows us to write the fundamental solution of the Helmholtz equation in Bloch form

$$
\psi\left(\mathbf{x}_{\perp}+\mathbf{T}\right)=\psi\left(\mathbf{x}_{\perp}\right) e^{i \mathbf{k}_{\perp} \cdot \mathbf{T}}
$$

where $\mathbf{T}$ is any vector of $\mathbf{T}_{\mathrm{mn}}, \mathbf{k}_{\perp}=k_{x} \hat{\mathbf{e}}_{x}+k_{y} \hat{\mathbf{e}}_{y}$ is an arbitrary transverse wave number.

Eqs. (2) and (3) together with the boundary conditions define the eigenvalue problem of finding $\lambda^{2}=\omega^{2} / c^{2}-k_{z}^{2}$ as a function of $\mathbf{k}_{\perp}$. The eigenvalue problem is solved numerically with a newly developed PBGSS code, which employs the standard coordinate-space finite-difference method.

\footnotetext{
*E-mail:smirnova@psfc.mit.edu.
} 


\section{RESULTS OF EIGENMODES AND BAND GAPS CALCULATIONS}

In this section, we present the results of PBGSS calculations of the eigenfrequencies for $\mathrm{TE}$ and $\mathrm{TM}$ modes in the two-dimensional square and triangular lattices. For all the plots presented we set $k_{z}=0$, which obviously does not affect the generality of the results.

\subsection{TM modes}

Figure 2 shows the dispersion characteristics (Brillouin diagrams) for the TM modes. In Fig. 2, $a / b=0.2$ and for the square lattice a global band gap between the first and second modes can be seen. For the triangular lattice the band gap between the second and third modes is about to occur. The first and the second mode are intersecting and there is no band gap between them

To determine the global TM band gaps, we perform extensive computations over all possible $\mathbf{k}_{\perp}$. The results are shown in Fig. 3. First thing to note is that for the TM mode the zeroth-order global band gap exists below the first mode, that is, there is a cutoff frequency. The cutoff frequency exists even for very small conducting poles and goes to zero logarithmically as $a / b \rightarrow 0$ (which is illustrated in Fig. 3 with a dashed curve continuation of the calculated cutoff curve). Shown in Fig. 3(a) are five lowest-order global TM band gaps for the square lattice. The first-order global TM band gap occurs between the first and second lowest modes. Higher-order global TM band gaps occur between the third and fourth, fourth and fifth, and fifth and sixth modes. There is no global band gap between the second and third modes. Shown in Fig. 3(b) are three lowest-order global TM band gaps for the triangular lattice. There is no global bandgap between the first and second modes. The first-order global TM band gap occurs between the second and third modes and the second-order global band gap is between the sixth and seventh modes.

\subsection{TE modes}

Figure 4 shows the Brillouin diagrams for the TE modes. Two cases correspond to the square and triangular lattices. In Fig. $4, a / b=0.2$ and there are no global TE band gaps for either square or triangular lattices. This is different from the TM case where the first band gap occurs between the first and second modes for $a / b \geq 0.1$.

We have also calculated the global TE band gaps in both types of lattices. The results are shown in Fig. 5. For the square lattice (Fig. 5(a)), we found that the first global TE band gap occurs between the first and second modes. Unlike the first global TM band gap, the lower boundary of this band gap decreases with increasing $a / b$. The higher order band gap opens and then closes between the sixth and the seventh modes for even lower ratio of $a / b$. For triangular lattice, we found more global TE band gaps as shown in Fig. 5(b). All of these gaps tend to close with increasing $a / b$ except for the lowest one, which occurs between the second and third modes. There is a global TE band gap, between the third and fourth modes, which appears for the lower ratios of $a / b$ than those for the lowest global TE band gap. Another global TE band gaps is between the sixth and seventh modes. In contrast to the
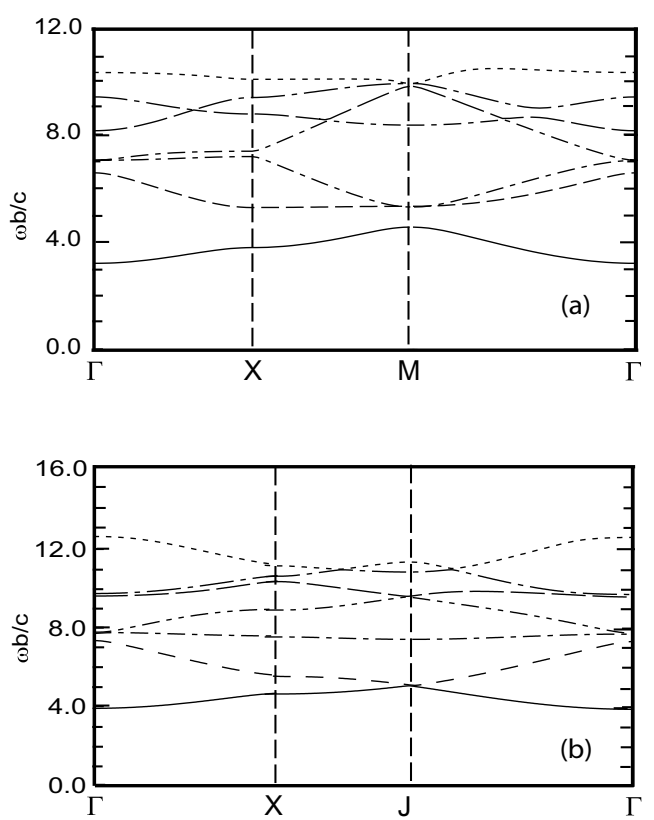

Figure 2: Seven lowest normalized eigenfrequencies for TM mode. Here $a / b=0.2$. The two cases correspond to (a) square lattice and (b) triangular lattice.
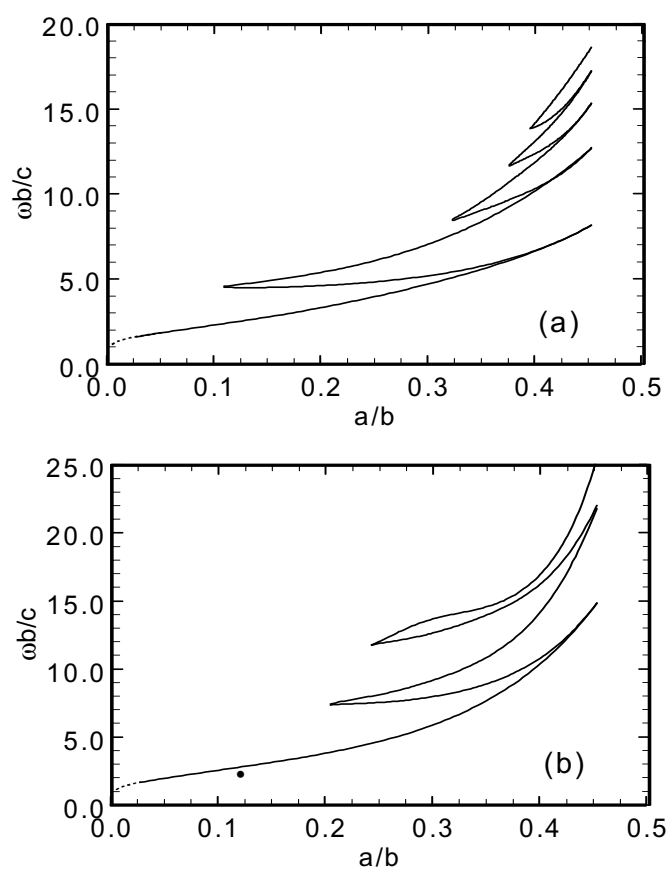

Figure 3: Plots of global frequency band gaps for TM mode as functions of $a / b$ for (a) square lattice and (b) triangular lattice. The solid dot represents the operating point of the $17 \mathrm{GHz}$ MIT accelerator cavity. 
TM mode, there is no cutoff in the case of TE mode. The first mode goes to zero at $\Gamma$ point for both square and triangular lattices. The absence of cutoff and closure of global TE band gaps with increasing $a / b$ resemble the behavior of the global band gaps in the lattice of vacuum poles in dielectric [2].
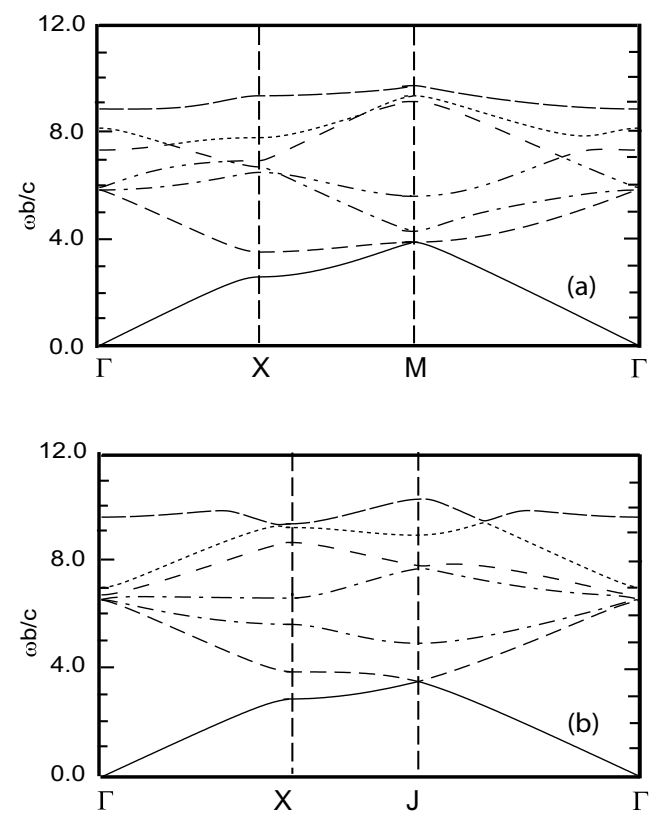

Figure 4: Seven lowest normalized eigenfrequencies for TE mode. Here $a / b=0.2$. The two cases correspond to (a) square lattice and (b) triangular lattice.
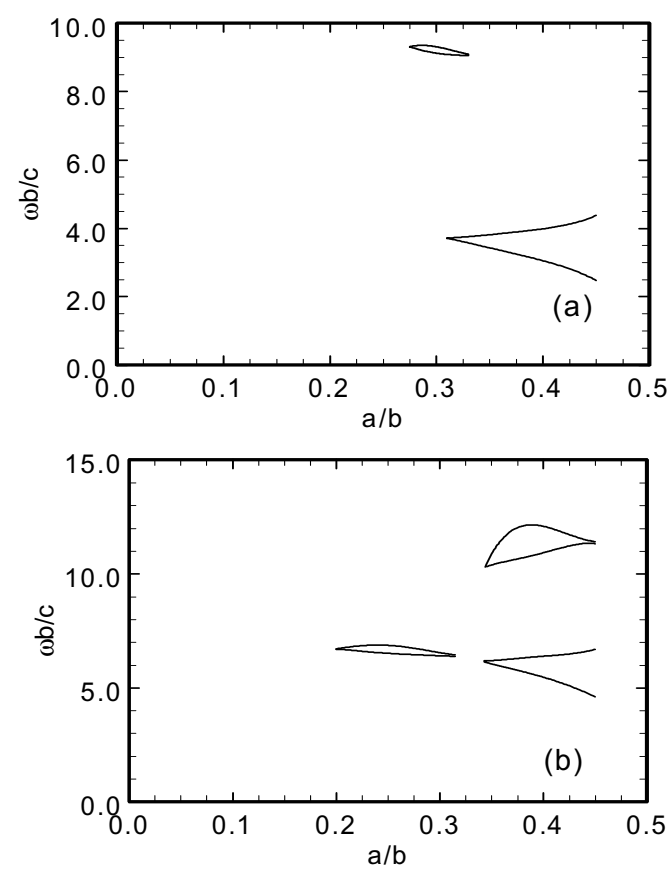

Figure 5: Plots of global frequency band gaps for TE mode as functions of $a / b$ for (a) square lattice and (b) triangular lattice.

\section{ACCELERATOR APPLICATIONS OF PBG STRUCTURES}

The results of the global bandgap calculations are useful for the PBG accelerator cavity design [6]. The PBG accelerator experiment was performed recently at MIT [7]. The accelerator cavity was made up of a triangular lattice of metal rods and operates in the TM mode at 17 GHz. A defect is created by one missing rode. The cavity has the pole radius $a=0.079 \mathrm{~cm}$ and the distance between poles $b=0.64 \mathrm{~cm}$, which corresponds to $a / b=1.23$ and $\omega b / c=2.28$. The cavity operational point is shown by the solid dot on Fig. 3(b). The cavity operates in the zero-order band gap (below the cutoff) and there are no other bandgaps above, so only one mode can be confined in the cavity, which solves the problem of wakefields. Fig.6 shows the cross-section of the HFSS model of the PBG accelerator cavity. The magnitude of the electric field of the confined mode is shown in color. The mode structure resembles the structure of the $\mathrm{TM}_{010}$ mode of conventional linac pillbox cavity.

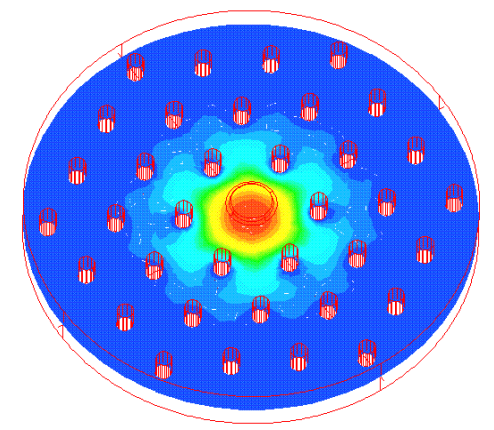

Figure 6: Magnitude of electric field in $\mathrm{TM}_{010}$-like mode in PBG cavity as obtained from the HFSS simulation.

\section{ACKNOWLEDGEMENT}

The work was supported by the DOE grant.

\section{REFERENCES}

[1] E.Yablonovitch, Phys. Rev. Lett. 58, p. 2059, 1987.

[2] J. D. Joannopoulos, R. D. Meade, and J. N. Winn, Photonic Crystals: Molding the Flow of Light (Princeton Univ. Press, Princeton, 1995).

[3] H.-Y.-D. Yang, IEEE Trans. MTT 44, p. 2688, 1996.

[4] N.A. Nicorovici, R.C. McPhedran, and L.C. Botten, Phys. Rev. E52, p. 1135, 1995.

[5] D.R.Smith, S. Schultz, N. Kroll, M. Sigalas, K. M. Ho, and C. M. Souloulis, Appl. Phys. Lett.65, p. 645, 1994.

[6] D.R. Smith, N. Kroll, and S. Schultz, in Advanced Accelerator Concepts, AIP Conference Proceedings 398, p. 761, AIP, New York, 1995.

[7] M.A. Shapiro, W.J. Brown, I. Mastovsky, J.R. Sirigiri, and R.J. Temkin, Phys. Rev. Special Topics: Accelerators and Beams 4, p. 042001, 2001.

[8] Ansoft High Frequency Structure Simulator - User's Manual, Ansoft Corp, 1999.

[9] E.I. Smirnova, C. Chen, "Global photonic band gaps in two-dimensional metallic lattices," Phys. Rev. Lett., Submitted for publication (May 2001). 